\title{
A low-dose, 6-week bovine colostrum supplementation maintains performance and attenuates inflammatory indices following a Loughborough Intermittent Shuttle Test in soccer players
}

\author{
Yiannis Kotsis $^{1} \cdot$ Anastasia Mikellidi $^{1} \cdot$ Cleopatra Aresti $^{1} \cdot$ Eleni Persia $^{1} \cdot$ \\ Aristomenis Sotiropoulos ${ }^{2} \cdot$ Demosthenes B. Panagiotakos $^{1}$. \\ Smaragdi Antonopoulou ${ }^{1}$ Tzortzis Nomikos ${ }^{1} \mathbb{C}$
}

Received: 8 October 2016 / Accepted: 10 February 2017 / Published online: 11 March 2017

(c) The Author(s) 2017. This article is published with open access at Springerlink.com

\begin{abstract}
Purpose The aim of the study was to investigate the effect of a 6-week, low-dose bovine colostrum (BC) supplementation on exercise-induced muscle damage (EIMD) and performance decline in soccer players following the Loughborough Intermittent Shuttle Test (LIST) during a competitive season period.

Methods In a double-blind, randomized, placebo-controlled design, two groups of soccer players were allocated to a $3.2 \mathrm{~g} /$ day of whey protein (WP, $N=8)$ or $\mathrm{BC}(N=10)$ and performed a pre- and a post-supplementation LIST. Maximum isometric voluntary contraction, squat jump (SQJ), countermovement jump, muscle soreness, blood cell counts, creatine kinase (CK), C-reactive protein (CRP) and interleukin-6 (IL-6) were monitored for 2, 24, 48, $72 \mathrm{~h}$ post-LIST.
\end{abstract}

The study was investigator initiated and the sponsor had no involvement in the design and conduct of the study, in the collection, management analysis and interpretation of the data or in the preparation, review or approval of the manuscript. The corresponding author had full access to all the data in the study and had the final responsibility for the decision to submit for publication.

Electronic supplementary material The online version of this article (doi:10.1007/s00394-017-1401-7) contains supplementary material, which is available to authorized users.

Tzortzis Nomikos

tnomikos@hua.gr

1 Department of Nutrition and Dietetics, Faculty of Health Sciences and Education, Harokopio University, Eleftheriou Venizelou 70, 17671 Athens, Greece

2 Faculty of Physical Education and Sport Sciences, National and Kapodistrian University of Athens, Athens, Greece
Results LIST induced transient increases in leukocytes, granulocytes, CK, muscle soreness, CRP, IL-6 and declines in lymphocytes and performance indices. Supplementation resulted in a faster recovery of SQJ, CK and CRP compared to pre-supplementation kinetics (trial $\times$ time: $p=0.001,0.056,0.014$, respectively) and lower incremental area under the curve (iAUC) for IL-6, only in the BC group [pre-: 31.1 (6.78-46.9), post-: 14.0 (-0.16 to 23.5) $\mathrm{pg} \mathrm{h} / \mathrm{ml}, p=0.034]$. Direct comparison of the two groups after supplementation demonstrated higher iAUC of SQJ [WP: $-195.2(-229.0$ to $(-52.5)), \mathrm{BC}:-15.8(-93.2$ to 16.8$) \mathrm{cm} \mathrm{h}, p=0.034$ ], a trend for lower iAUC of CK in the BC group [WP: 18,785 (4651-41,357), BC: 8842 $(4807-14,802) \mathrm{U} \mathrm{h} / \mathrm{L}, p=0.081]$ and a significant intervention $\times$ time interaction for CRP $(p=0.038)$ in favor of BC.

Conclusions Post-exercise EIMD may be reduced and performance better maintained by a low dose of BC administration following LIST in soccer players.

Keywords Bovine colostrum - Exercise-induced muscle damage $\cdot$ Loughborough Intermittent Shuttle Test - Soccer · Inflammation $\cdot$ Squat jump

$\begin{array}{ll}\text { Abbreviations } \\ \text { iAUCs } & \text { Incremental area under the curve } \\ \text { BC } & \text { Bovine colostrum } \\ \text { CK } & \text { Creatine kinase } \\ \text { CMJ } & \text { Counter movement jump } \\ \text { CRP } & \text { C-reactive protein } \\ \text { EIMD } & \text { Exercise-induced muscle damage } \\ \text { IL-6 } & \text { Interleukin-6 } \\ \text { LIST } & \text { Loughborough Intermittent Shuttle Test } \\ \text { MIVC } & \text { Maximum isometric voluntary contraction } \\ \text { SQJ } & \text { Squat jump }\end{array}$

Abbreviations

iAUCs Incremental area under the curve

BC Bovine colostrum

CK Creatine kinase

CMJ Counter movement jump

CRP C-reactive protein

EIMD Exercise-induced muscle damage

IL-6 Interleukin-6

LIST Loughborough Intermittent Shuttle Test

MIVC Maximum isometric voluntary contraction

SQJ Squat jump 
VAS Visual analog scale

WP Whey protein

\section{Introduction}

Elite-level soccer games are characterized by high-intensity intermittent physical activity. The repetitive and eccentric nature of soccer movements (accelerations, decelerations, kicking, jumping, and tackling) can cause muscle damage, accompanied by the leakage of muscle enzymes and other functional proteins in the circulation, oxidative stress, inflammation and decline in muscle performance $[1,2]$. The clinical phenotype of post-match muscle damage can strongly impair the recovery process [3]. Taking into account the congested schedule of elite soccer players ( 2 or even 3 matches per week) the recovery period is not optimal for the restoration of pre-match exercise capacity and performance, while it may increase the risk for muscle injuries [4].

Since the extent of muscle damage can determine the duration of the recovery period, several strategies, aiming to attenuate muscle damage and accelerate the recovery period, are under research. However, the majority is adopting physiotherapeutic and training modalities like stretching, cryotherapy, active recovery, sleeping, massage, electrical stimulation and compression garments [5], while the nutritional or supplementation interventions are much less studied under real post-soccer match conditions. Interventions including the administration of creatine [6], antioxidant vitamins [7, 8], astaxanthin [9], beta-alanine [10], omega-3 fatty acids [11] are tested for their ability to reduce exercise-induced muscle damage (EIMD) or to increase exercise performance after a soccer match or a simulation of a soccer match. However, the results so far are inconclusive concerning the ability of supplements to improve the recovery process [5].

Bovine colostrum (BC) is the first milk produced by the mammary glands of the mother during the final days of pregnancy and the first days postpartum (1-4 days). It is as rich in carbohydrates, proteins, fats and micro-nutrients (minerals, trace elements) as the regular milk, but it additionally contains high amounts of oligosaccharides, growth factors, antimicrobial agents and immunoregulatory compounds [12]. It is therefore a natural multi-ingredient supplement with potential pleiotropic properties. Previous studies focused, mainly, on its effect on exercise performance, body composition [13], immune system and its ability to counteract upper respiratory tract infections [14]. However, the doses of $\mathrm{BC}$ in these studies were relatively high (20-60 g/day). So far, the ability of BC to enhance the recovery after a high-intensity soccer match is implied only indirectly through its anabolic properties [15], its buffering capacity [16] and its ability to attenuate post-exercise intestinal permeability [17] and oxidative stress [18]. In order to shed more light on this issue, aim of the present study was to investigate the effect of a low-dose BC supplementation on EIMD and performance in soccer players, during their competitive season, following the Loughborough Intermittent Shuttle Test (LIST) which simulates the activity pattern of a real soccer match [19].

\section{Materials and methods}

\section{Study design}

The study had a double-blind, randomized, placebo-controlled, parallel group, design. Participants initially completed a pre-supplementation LIST (LIST 1) and muscle damage indices were monitored for $72 \mathrm{~h}$ post-exercise. Then, they randomly allocated to the whey protein (WP) or BC group, received the respective supplements for 6 weeks and repeated the LIST protocol (LIST 2) by the end of the intervention period. The muscle damage and performance indices were again monitored for $72 \mathrm{~h}$.

A week before LIST 1 subjects visit the laboratory for the preliminary screening, cardiopulmonary assessment and familiarization of all experimental procedures and LIST protocol. On the day of LIST 1, players arrived at the laboratory between 07:00 and 08:00 am after an overnight fast. A resting blood sample was taken and muscle strength, jumping performance, perceived muscle soreness and body composition were assessed. Afterwards, subjects consumed a standard meal consisting of two whole grain bread slices, a slice of smoked turkey fillet, $30 \mathrm{~g}$ of yellow Swiss cheese and a $240 \mathrm{ml}$ of fresh orange juice (giving approximately $397 \mathrm{kcal} ; 59 \mathrm{~g}$ carbohydrates, $11 \mathrm{~g}$ fat, $15 \mathrm{~g}$ protein). EIMD biochemical markers, through venous blood samples, muscle performance and perceived muscle pain were evaluated 2, 24, 48 and $72 \mathrm{~h}$ post-LIST. Participants who successfully completed LIST 1 were randomly allocated to WP or BC group and received $3.2 \mathrm{~g} /$ day of $\mathrm{WP}$ or $\mathrm{BC}$, respectively, for 6 weeks. By the end of the supplementation period all volunteers repeated the experimental protocol (LIST 2) and evaluations with the same order and time intervals.

The experimental protocol took place during the competitive period of the soccer season. During the whole protocol period, subjects were instructed to keep their usual eating habits and to restrain from any additional vitamin, antioxidant and performance-enhancing dietary supplementation or recovery treatment. Compliance to the protocol was checked with 24-h recalls. Subjects were also instructed to abstain from exhaustive exercise for 72-h preand post-LISTs and followed a standardized training regimen. During the whole supplementation period, with the 
exception of the 72-h pre- and post-LIST periods, the players were engaged in their habitual soccer training routine that included 4-5 training sessions and one official match per week. A detailed depiction of the experimental design is shown in Fig. 1.

\section{Ethics}

The protocol was approved by the Ethical Committee of Harokopio University. All procedures performed in studies involving human participants were in accordance with the ethical standards of the institutional and/or national research committee and with the 1964 Helsinki declaration and its later amendments or comparable ethical standards. Informed consent was obtained from all individual participants included in the study.

\section{Subjects}

For the purposes of the study 25 soccer players from the third and fourth division of the Greek National league were initially recruited (Fig. 2). Exclusion criteria were medical treatment, history of cardiovascular or any other inflammatory disease, cold or flu, acute respiratory infection, dental problems, renal/hepatic abnormalities, lactose intolerance and recent history of muscle injuries. Three of them were excluded from the study either because they did not meet the inclusion criteria or they declined participation. The rest of them $(N=22)$ completed the pre-supplementation LIST (LIST 1) and allocated to either the placebo group (WP, $N=11$ ) or the bovine colostrum group (BC, $N=11$ ). Three volunteers from the WP group and one volunteer from the BC group could not complete the intervention protocol due to upper respiratory tract infection and muscle strain. At the end, eight volunteers of the WP group and ten volunteers of the BC group completed the post-supplementation LIST (LIST 2) (Fig. 2). The WP group consisted of one defender, five midfielders, one full back/winger and one central attacker while $\mathrm{BC}$ group consisted of four defenders, four midfielders, one full back/winger and one central attacker.

\section{Sample size determination}

To evaluate standardized differences in CK levels equal to $20 \%$, a sample size of 10 participants per each group was considered adequate to achieve statistical power $>0.90$ at significance level of $p<0.05$ of two-sided hypotheses.

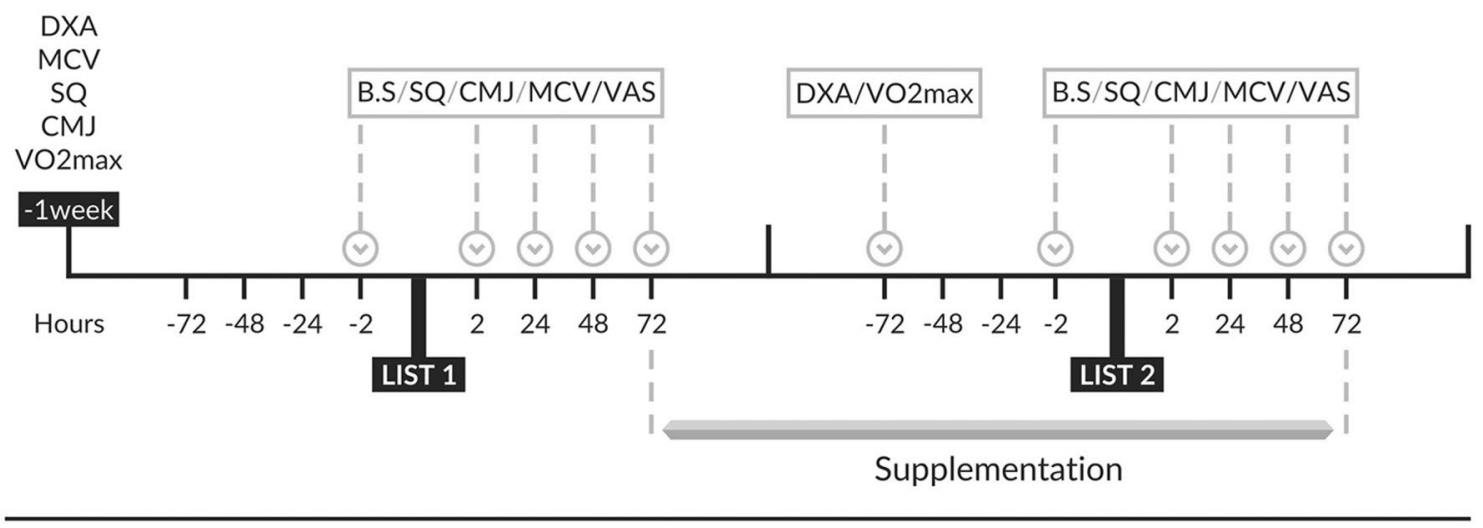

LIST (simulation of a soccer match)

set 1. Recovery (3min) set 2 Recovery (3min) set 3 Recovery (3min) set 4 Recovery ( $3 \mathrm{~min}$ ) set 5 Recovery (3min) 1
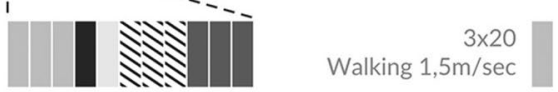

$1 \times 20$
Sprinting

$4 \mathrm{sec}$

$3 \times 20$ Cruising @ 55\% VO2max

$\leqslant \quad 3 \times 20$ Running

Modified FIFA 11 program: Basic running exercises (low intensity run forward \& backward, hip in and out, quick forward and static core). No additional eccentric exercises used (i.e hamstrings, jumping)

-72 hours: 15 min of modified FIFA 11 warm up, 20 min of technical exercises, passing games/crossing, 20 min tactical positioning (offending/defending), Recovery: Jogging and light self-administered stretching.

-48 hours: 12 min of FIFA 11 warm up, 20 min of 5vs2, Sprinting: 4 set of 5x10, Recovery: Jogging and light self-administered stretching.

-24 hours: Day Off

+24 hours: Day off or $2 \times 8$ min of jogging at $50-60 \%$ of their HRmax and light self-administered stretching.

+48 hours: Day off or $2 \times 8$ min of jogging at $50-60 \%$ of their HRmax and light self-administered stretching.

+72 hours: 15 min of FIFA 11 warm up, 20 min of technical exercises, passing games, 2 sets of 10 runs (box to box) at $80 \%$ Hrmax, Recovery: Jogging and light self-administered stretching.

Fig. 1 Schematic representation of the experimental protocol 
Fig. 2 Flowchart of volunteers' recruitment

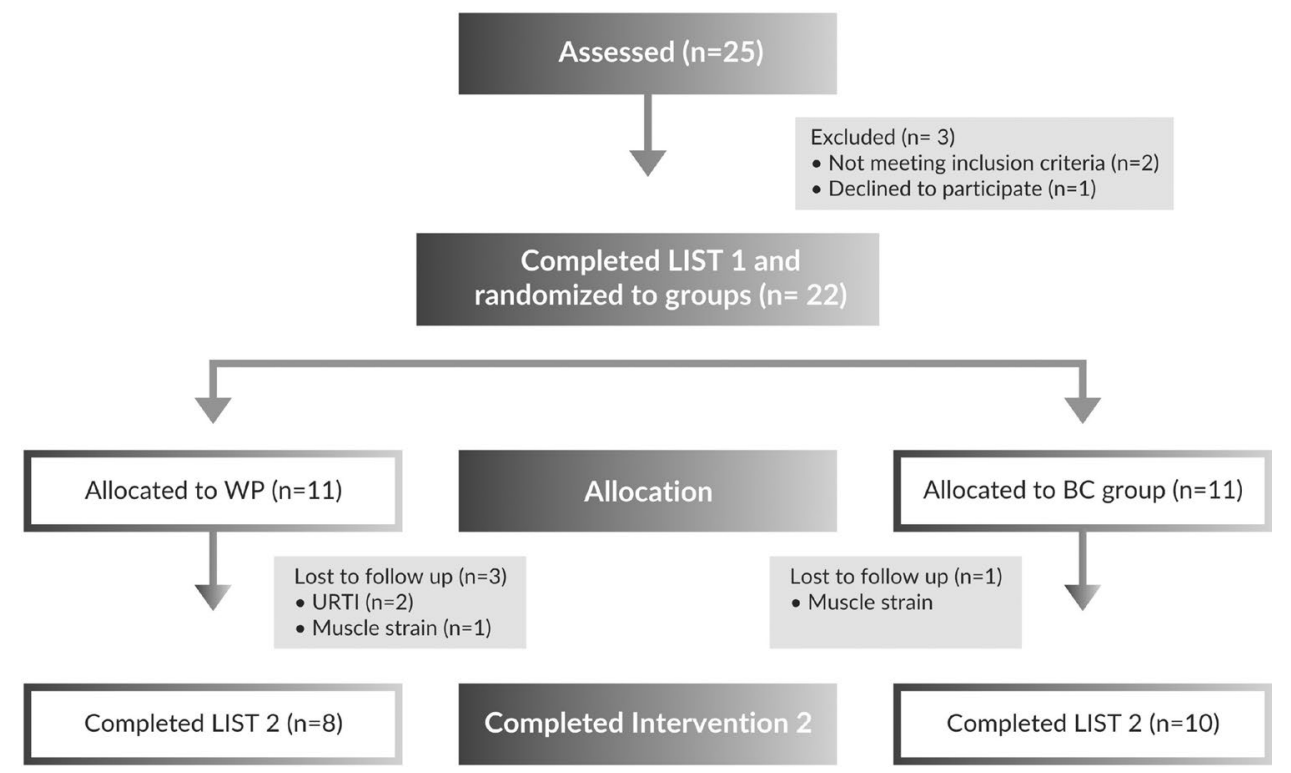

Despite the fact that we finished with less subjects in the WP group $(n=8)$, the working sample was also adequate to achieve statistical power equal to $90 \%$ having the aforementioned conditions.

\section{Randomization}

A random sequence of numbers (i.e., 01001...) was created by an algorithm in MS Excel. Participants were allocated to $\mathrm{BC}$ (if 0 ) or WP (if 1).

\section{Supplementation}

Participants of the BC group received $3.2 \mathrm{~g} /$ day (8 capsules $\times 400 \mathrm{mg}$ of $\mathrm{BC}$ per capsule) of a commercially available bovine colostrum containing $378 \mathrm{kcal}, 67 \mathrm{~g}$ protein, $17 \mathrm{~g}$ carbohydrates and $4.7 \mathrm{~g}$ fat per $100 \mathrm{~g}$ (Colostrum Compact, LR Health and Beauty Systems, Germany) while the WP group received $3.2 \mathrm{~g} /$ day of commercially available whey protein $(8$ capsules $\times 400 \mathrm{mg}$ of WP per capsule) without flavoring and sweeteners (Ener Zona Proteine Whey $90 \%$, Italy) containing $369 \mathrm{kcal}, 90 \mathrm{~g}$ protein, $1 \mathrm{~g}$ carbohydrates and $0.5 \mathrm{~g}$ fat per $100 \mathrm{~g}$. The whey protein was encapsulated in capsules, provided by LR, which were identical with the ones containing BC. The participants were advised to consume 4 capsules twice per day, half an hour before breakfast and dinner and were given 3 identical 56-capsule boxes containing either BC or WP, enough for a specific supplementation period of 3 weeks. By the end of the third week subjects were given the last four capsule boxes containing enough capsules for the remaining 3 weeks plus the 4 days of LIST 2 . Then they were asked to record their daily supplementation consumption and bring back the boxes with the remaining capsules inside them. A 85 and $82 \%$ adherence was recorded for the WP and BC group, respectively.

\section{Loughborough Intermittent Shuttle Test}

The 90-min shuttle run test was conducted according to Thompson et al. in an artificial surface soccer pitch [20]. Volunteers were required to run between two lines, $20 \mathrm{~m}$ apart, at various speeds dictated by an audio signal based on the velocities corresponding to their individual 55 and $95 \%$ of $\mathrm{VO}_{2 \max }$. The protocol consisted of 5 blocks of continuous running separated by $3 \mathrm{~min}$ of passive recovery for a total duration of $90 \mathrm{~min}$. Heart rate was recorded via telemetry at 5-s intervals (Polar Team2 Pro, Polar, Finland) and averaged for each set (Fig. 1).

\section{Anthropometric and body composition measurements}

Body weight and standing height were measured in light clothing, without shoes using a digital scale (Seca 861; Hamburg, Germany) with an accuracy of $0.1 \mathrm{~kg}$ and a stadiometer (Seca Leicester Height Measure; Seca, Vogel $\&$ Halke) to the nearest $0.1 \mathrm{~cm}$. Body composition was assessed by DXA (Lunar, Corporation, Brussels, Belgium) set at medium speed and according to the manufacturer's instructions [21].

\section{Nutritional assessment}

Dietary intake before supplementation was assessed by three 24-h recalls which were collected the week before LIST 1 (two weekdays and one weekend). Similarly, dietary 
intake after supplementation was assessed by three more 24-h recalls, collected the week before LIST 2 . The type of foods consumed (e.g., in terms of fat-content, brand name, constituents of mixed dishes, etc.), and the quantities or volumes were recorded in detail, using common household or other measures. Data from recalls were analyzed for their energy, macro- and micronutrient content by the Nutritionist Pro, version 2.2 software (Axxya Systems-Nutritionist Pro, Stafford, TX, USA), using a hand-coding procedure. The Nutritionist Pro food database was expanded by adding analyses of traditional Greek food and recipes.

\section{Cardiopulmonary assessment}

The players performed an incremental $(0.5 \mathrm{~km} / \mathrm{h}$ increase each 1 min step) treadmill (Technogym Runrace, Gambettola, Italy) test until voluntary exhaustion to determine maximal oxygen uptake $\left(V \mathrm{O}_{2 \max }\right)$, maximal heart rate (Polar Electro, Finland) and running speeds corresponding to 55 and $95 \%$ of $V \mathrm{O}_{2 \max }$. Expired respiratory gas fractions were measured using an open circuit breath-by-breath automated gas-analysis system (Vmax 229D, Sensormedics, Yorba Linda, CA).

\section{Maximum isometric voluntary contraction (MIVC)}

During their first visit at the laboratory all subjects performed a specific submaximal protocol on the isokinetic dynamometer (BIODEX System 3 Pro, NY, USA) to familiarize with the isokinetic device and test procedure. Before muscle function measurements, subjects perform a standardized warm-up consisting of 5-min period on a cycle ergometer (Monark E-824) with a fixed load corresponding to $2 \%$ of body weight. MIVC of the knee extensors was measured at a knee joint angle of $90^{\circ}\left(0^{\circ}=\right.$ full knee extension). The subjects were instructed to kick as hard and fast as they could for five maximal repetitions lasting $5 \mathrm{~s}$ separated by $60 \mathrm{~s}$ of rest. The three MIVC trials were averaged together to give the score for that particular measurement period.

\section{Jump performance}

Squat jump (SQJ) and countermovemt jump (CMJ) tests were conducted using a photocell device (Optojump, Microgate, Italy) according to Bosco et al. [22]. Subjects warmed up for $5 \mathrm{~min}$ of on a stationary bicycle and performed 2 sets of 6 reps of submaximal SQJ and CMJ jumps by bending the knees to a preferred starting push-off position for CMJ and from a fixed knee bent at $90^{\circ}$ for SQJ. For both jumping techniques hands were placed on hips. Then 3 maximal jumps were executed for each technique. A minimum of 2-min rest was allowed between jump trials.
The highest vertical jump (cm) and the longest flying time (s) were registered and used for data analyses.

\section{Perceived muscle soreness (PMS)}

Subjects self-reported muscle soreness by performing 3 full squat movements and passive stretching of specific muscles (quadriceps, hamstrings, abductors, adductors and gastrocnemius). We used a visual analog scale (VAS) from 0 (absence of soreness) to 10 (extremely sore) and a visual hologram of human muscles [20] where subjects pointed at the specific muscle area and rated the soreness.

\section{Blood sampling and biochemical assays}

Venous blood samples were obtained from each subject. Complete blood count was determined in EDTA anticoagulated whole blood with a Mindray BC-3000 hematology analyzer (Mindray, Shenzhen, P.R. China). The activity of creatine kinase (CK) was determined spectrophotometrically by a commercially available kit (Biosis, Athens, Greece) modified for 96-well plates according to manufacturer's instructions. High-sensitivity CRP and IL-6 were determined in serum by commercially available ELISA kits (Quantikine, R\&D Systems, Abingdon, UK).

\section{Statistical analysis}

Quantitative variables are reported as median with interquartile range (25th percentile-75th percentile) and qualitative, as frequencies. In order to estimate the response of each variable across the whole recovery period incremental, areas under the characteristic curve (iAUCs) were calculated for each variable by the trapezoid rule. Due to the small sample size and the skewed distribution we used the non-parametric Mann-Whitney test to compare: (a) the pre-LIST (baseline) values of WP and BC pre-supplementation and post-supplementation and (b) the iAUCs of WP and $\mathrm{BC}$ groups' pre-supplementation and post-supplementation. A non-parametric analysis for repeated measures (Friedman test) was also applied to identify significant changes of VAS indices over time. Post hoc analysis with Wilcoxon signed-rank tests was conducted with a Bonferroni correction applied due to multiple comparisons, resulting in a significance level set at $p<0.01$. Comparison of the response curves (WP LIST 1 vs WP LIST 2, BC LIST 1 vs BC LIST 2, WP LIST 1 vs BC LIST 1 and WP LIST 2 vs BC LIST 2) were performed using a two-way (trial $\times$ time) repeated-measures ANOVA, using log-transformed variables to reduce non-uniformity of error. ANOVA models incorporated a Greenhouse-Geisser correction for multisample asphericity. Any significant main effects identified in the ANOVA were further analyzed for post hoc paired 
t tests with Holm-Bonferroni correction. All statistical calculations were made with SPSS version 21.0 (Statistical Package for Social Sciences, SPSS, Chicago, Illinois, USA).

\section{Results}

The WP and BC groups shared similar anthropometric, training, performance, hematological and biochemical preexercise characteristics that did not change after the 6-week supplementation period (Table 1). Dietary analysis did not reveal any significant differences in macronutrient intake between LIST 1 and LIST 2, neither for the WP group, nor for the BC group. In detail, energy intake for WP group was $2633 \pm 472$ vs $2711 \pm 728 \mathrm{kcal}$ (protein: $17.8 \pm 3.7 \mathrm{vs}$ $18.3 \pm 3.6 \%$, carbohydrates: $44 \pm 8.4$ vs $38.9 \pm 7.8 \%$, fat: $38.9 \pm 8.9$ vs $38.1 \pm 5.6 \%)$ and for BC group $2676 \pm 562$ vs $2582 \pm 372 \mathrm{kcal}$ (protein: $19.3 \pm 3.4$ vs $18.2 \pm 5.1 \%$, carbohydrates: $43 \pm 7.4$ vs $43.9 \pm 71.6 \%$, fat: $36.4 \pm 5.4$ vs $38.6 \pm 56.6 \%$ ) for LIST 1 vs LIST 2 , respectively. No differences between the dietary intake of WP and BC were also observed before LIST 1 or LIST 2.

The pre-supplementation kinetics of the measured variables (LIST 1) demonstrated either transient or sustained changes from baseline values in both groups indicating the ability of LIST to induce post-exercise hematological, biochemical and performance alterations (Tables 2, 3; Figs. 3, 4, 5, 6). Specifically, an acute but transient, twofold increase, of $\mathrm{WBC}$ is observed $2 \mathrm{~h}$ post-LIST 1 . The rapid elevation of circulating WBC is attributed to a $\sim 2.5$ fold increase of granulocytes although a significant $30 \%$ decline of lymphocytes was also observed at the same time point. The leukocyte levels returned to baseline values at $24 \mathrm{~h}$ and remained there up to $72 \mathrm{~h}$ post-LIST 1 . Concerning the exercise performance indices, a sustained significant decline of all markers (MIVC, SQJ and CMJ) was observed from 2 to $48 \mathrm{~h}$ post-LIST 1 . MIVC declined $\sim 8$ to $10 \%$ from 2 to $48 \mathrm{~h}, \mathrm{CMJ}$ declined $\sim 8$ to $10 \%$ from 24 to $48 \mathrm{~h}$ while a 7-9\% decline of SQJ was observed at 24-48 h. Moreover, a significant increase of perceived muscle pain in all muscle groups was evident, mainly at $24-48 \mathrm{~h}$. The greater increase was found in knee extension and flexion muscles. Finally, CK values started to elevate 2 -h postLIST 1 but peaked at 24 or $48 \mathrm{~h}$ depending on the volunteer (3- to 4-fold increase). A delayed increase, at 24-48 h (fivefold), was demonstrated for CRP while the increase of IL- 6 was acute showing a fourfold peak at $2 \mathrm{~h}$ post-exercise and a return to basal levels at $24 \mathrm{~h}$. The comparison of the iAUCs of all measured variables after LIST 1 demonstrated no significant differences between WP and BC groups indicating that the response to the LIST protocol, for all measured EIMD variables, was similar between the two groups (data not shown).
Table 1 Baseline, pre-LIST, characteristics of volunteers in the WP and $\mathrm{BC}$ groups

\begin{tabular}{|c|c|c|c|c|}
\hline & \multicolumn{2}{|l|}{ LIST 1} & \multicolumn{2}{|l|}{ LIST 2} \\
\hline & WP & $\mathrm{BC}$ & WP & $\mathrm{BC}$ \\
\hline Age (years) & $21.5(19.5-22.2)$ & $22.0(19.5-23.2)$ & $21.5(19.5-22.2)$ & $22.0(19.5-23.2)$ \\
\hline BMI $\left(\mathrm{kg} / \mathrm{m}^{2}\right)$ & $22.9(22.2-23.4)$ & $23.6(22.8-24.1)$ & $23.0(22.3-23.6)$ & $23.3(22.8-23.9)$ \\
\hline Training (min/week) & $505(397-637)$ & $570(417-600)$ & $520(367-621)$ & $552(400-588)$ \\
\hline Total fat (\% of BW) & $13.4(10.8-15.2)$ & $14.1(10.5-14.4)$ & $13.4(11.0-14.7)$ & $14.7(11.4-15.9)$ \\
\hline$V \mathrm{O}_{2 \max }(\mathrm{mL} / \mathrm{kg} / \mathrm{min})$ & $51.4(49.5-54.3)$ & $53.7(51.8-54.3)$ & $51.0(49.2-54.0)$ & $52.3(50.4-54.1)$ \\
\hline MI VC (Nm) & $321(269-337)$ & $349(274-375)$ & $315(242-374)$ & $299(260-365)$ \\
\hline $\mathrm{CMJ}(\mathrm{cm})$ & $34.1(31.3-37.8)$ & $36.5(32.3-38.6)$ & $33.6(32.8-38.0)$ & $34.8(32.7-37.5)$ \\
\hline $\mathrm{SQJ}(\mathrm{cm})$ & $30.8(29.4-35.4)$ & $34.3(30.4-36.6)$ & $32.0(30.1-36.4)$ & $33.1(29.5-34.6)$ \\
\hline White blood cells $\left(10^{3} / \mu \mathrm{L}\right)$ & $6.2(5.0-6.9)$ & $5.5(4.9-5.9)$ & $6.2(5.7-6.8)$ & $5.3(4.8-6.3)$ \\
\hline Lymphocytes $\left(10^{3} / \mu \mathrm{L}\right)$ & $2.2(2.0-2.6)$ & $2.2(1.9-2.4)$ & $2.5(2.0-2.8)$ & $2.4(1.8-2.6)$ \\
\hline Neutrophils $\left(10^{3} / \mu \mathrm{L}\right)$ & $3.5(2.3-4.2)$ & $2.6(2.4-3.5)$ & $3.6(3.1-6.1)$ & $2.9(2.2-4.2)$ \\
\hline Erythrocytes $\left(10^{6} / \mu \mathrm{L}\right)$ & $5.19(4.92-5.44)$ & $5.06(4.65-5.77)$ & $5.02(4.87-5.39)$ & $5.13(4.71-5.47)$ \\
\hline Hemoglobin $(\mathrm{g} / \mathrm{dL})$ & $15.3(14.4-16.6)$ & $15.5(14.3-15.9)$ & $15(14.1-15.6)$ & $14.7(13.9-15.05)$ \\
\hline Platelets $\left(10^{3} / \mu \mathrm{L}\right)$ & $222(199-249)$ & $226(192-238)$ & $210(166-286)$ & $199(180-249)$ \\
\hline Creatine kinase (U/L) & $168(102-396)$ & $152(100-249)$ & $143(79-358)$ & $174(108-249)$ \\
\hline C-reactive protein (mg/dL) & $0.27(0.18-0.41)$ & $0.40(0.21-0.57)$ & $0.29(0.18-0.90)$ & $0.46(0.33-0.65)$ \\
\hline IL-6 (pg/mL) & $0.61(0.40-0.86)$ & $0.62(0.42-1.0)$ & $0.65(0.42-1.11)$ & $0.78(0.55-0.96)$ \\
\hline
\end{tabular}

Results are given as median (interquartile range). No significant differences were observed between the pre-LIST 1 values of WP and BC or between the pre-LIST 2 values of WP and BC (Mann-Whitney $U$ test). No significant differences were observed between the pre-LIST 1 and pre-LIST 2 values of WP group and between the pre-LIST 1 and pre-LIST 2 values of BC group (Wilcoxon signed-rank test) 


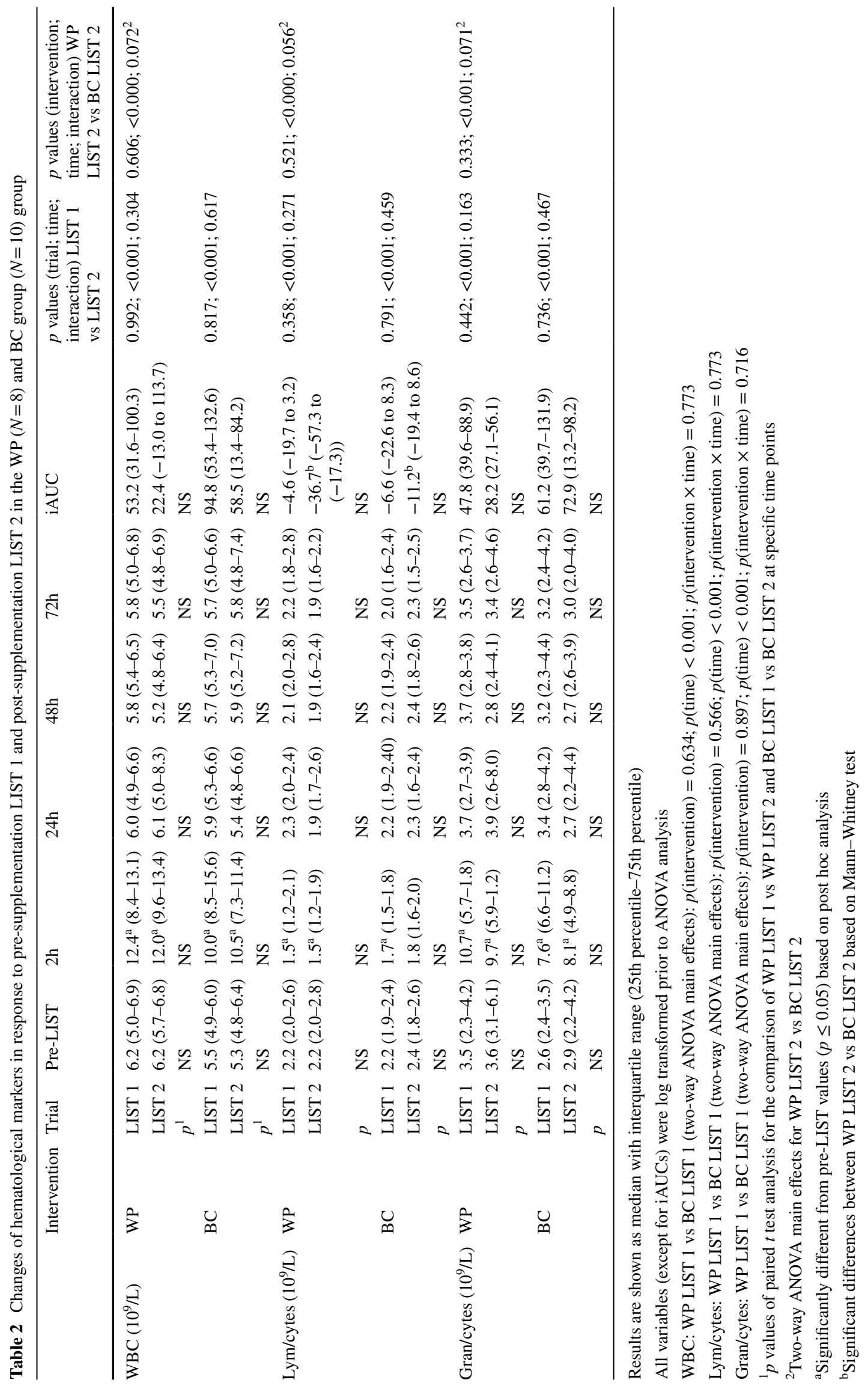




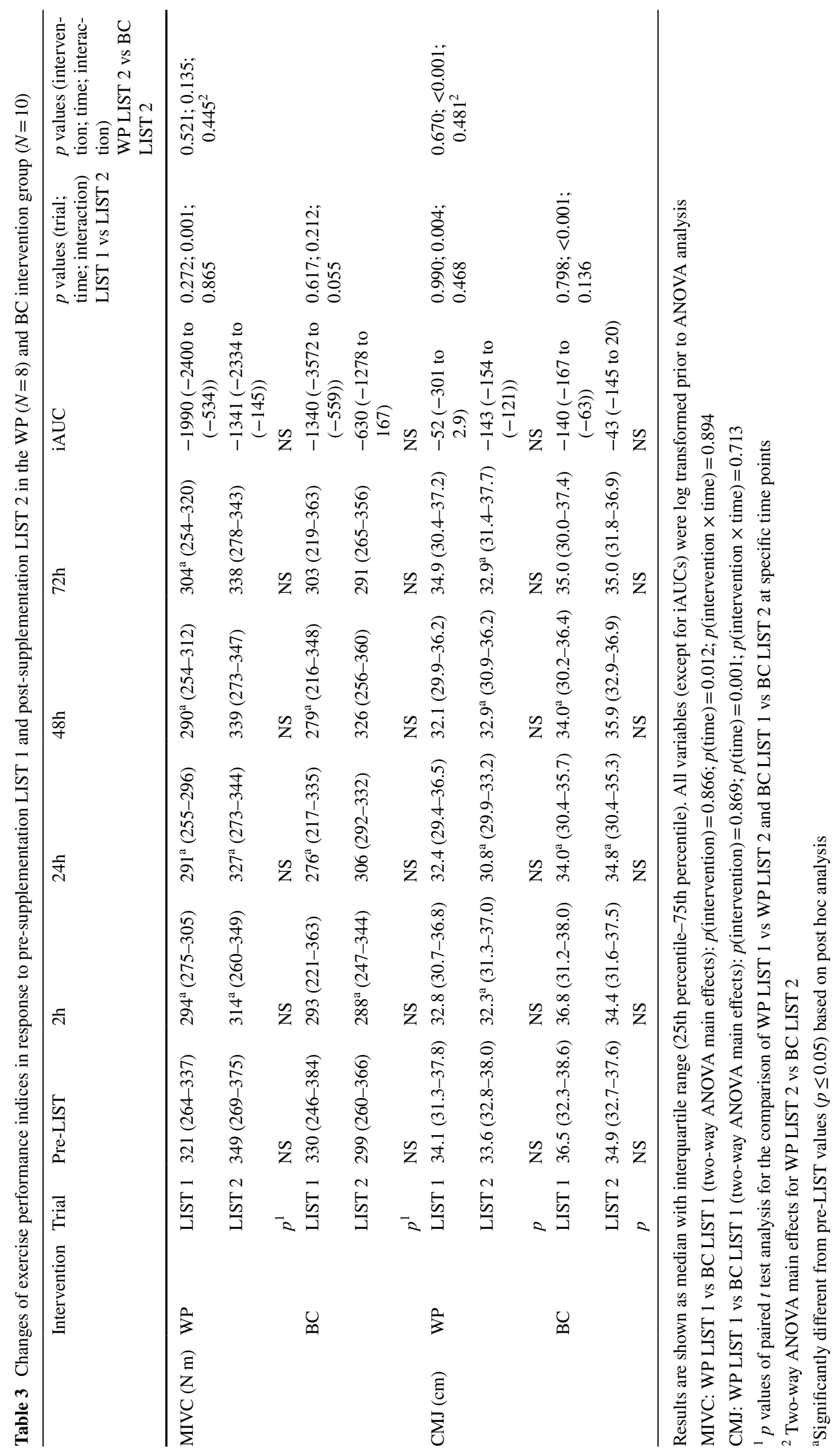



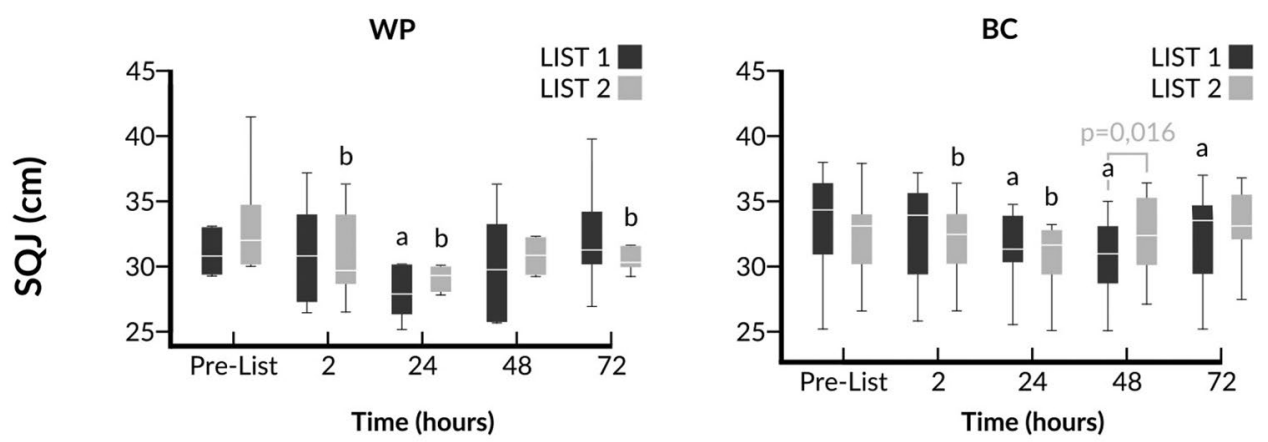

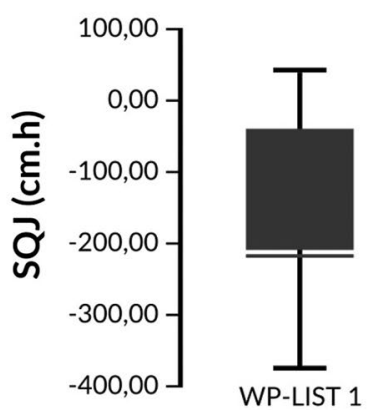

Fig. 3 Changes of SQJ and comparison of iAUCs in response to LIST pre- and post-supplementation. ${ }^{\mathrm{a}} p<0.05$ vs pre-LIST 1 values, ${ }^{\mathrm{b}} p<0.05$ vs pre-LIST 2 values. SQJ values were $\log$ transformed prior to ANOVA analysis. WP LIST 1 vs WP LIST 2 (twoway ANOVA main effects): $p$ (trial) $=0.751 ; p$ (time $) \leq 0.001$; $p($ trial $\times$ time) $=0.190$. BC LIST 1 vs BC LIST 2 (two-way

In order to assess whether the 6-week supplementation with either WP or BC was able to change the volunteers' response to the LIST protocol, we compared the kinetics of the measured variables pre- and post-supplementation (WP LIST 1 vs WP LIST 2 and BC LIST 1 vs BC LIST 2 and) for each group, separately. Specifically, we compared the absolute values and percent changes at each time point along with the iAUC of LIST 1 and LIST 2 for every volunteer in a pair-wise fashion. In addition, a direct comparison between groups was made before (WP LIST 1 vs BC LIST 1) and after supplementation (WP LIST 2 vs BC LIST 2).

Concerning the leukocyte counts no significant differences of WBC, granulocytes and lymphocyte kinetics were observed after LIST 2 compared to LIST 1, either in the WP or the BC group, indicating that neither WP nor BC supplementation were able to alter the leukocytes response to LIST (Table 2). Direct comparisons of WP vs BC before and after supplementation also showed no differences between groups in the exercise-induced changes of the hematological markers although a higher iAUC for lymphocytes $(p=0.017)$ was observed in the BC group compared to WP group after LIST 2 (Table 2).

Comparison of the VAS recordings at individual time points or as iAUC demonstrated no significant differences
ANOVA main effects): $p$ (trial) $=0.893 ; p$ (time) $<0.001$; $p($ trial $\times$ time) $=0.001$. WP LIST 1 vs BC LIST 1 (two-way ANOVA main effects): $p$ (intervention) $=0.680 ; p$ (time) $\leq 0.001$; $p($ intervention $\times$ time $)=0.207$. WP LIST 2 vs BC LIST 2 (twoway ANOVA main effects): $p$ (trial) $=0.819 ; \mathrm{p}($ time $) \leq 0.001$; $p($ trial $\times$ time $)=0.065$

of delayed-onset muscle soreness in none of the five muscle groups between LIST 1 and LIST 2 in both groups with the exception of lower VAS recordings of the knee flexors after post-supplementation LIST 2 in the WP group (Table 4, Supplemental material).

When the performance responses were compared, similar declines for MIVC and CMJ were observed for LIST 1 and LIST 2 in both groups (Table 3). However, a faster recovery of the SQJ performance was observed only in the $\mathrm{BC}$ group which was reflected in a significant trial $\times$ time interaction $(p=0.001)$ between BC LIST 1 and BC LIST 2, a significant intervention $\times$ time interaction between WP LIST 2 and BC LIST 2 and a better SQJ performance $48 \mathrm{~h}$ post-LIST 2 compared to post-LIST 1 in the BC group. Although absolute values could not show a significant trial effect and intervention effect, when ANOVA analysis was conducted with the \% changes of SQJ values (data not shown) significant differences were observed between BC LIST 1 and BC LIST 2 $(p$ trial $=0.048)$ and between WP LIST 2 and BC LIST 2 ( intervention $=0.010)$. In addition, less negative iAUCs were recorded for the nine out of ten volunteers in the BC group. This trend was not observed for the WP group (Fig. 3). 

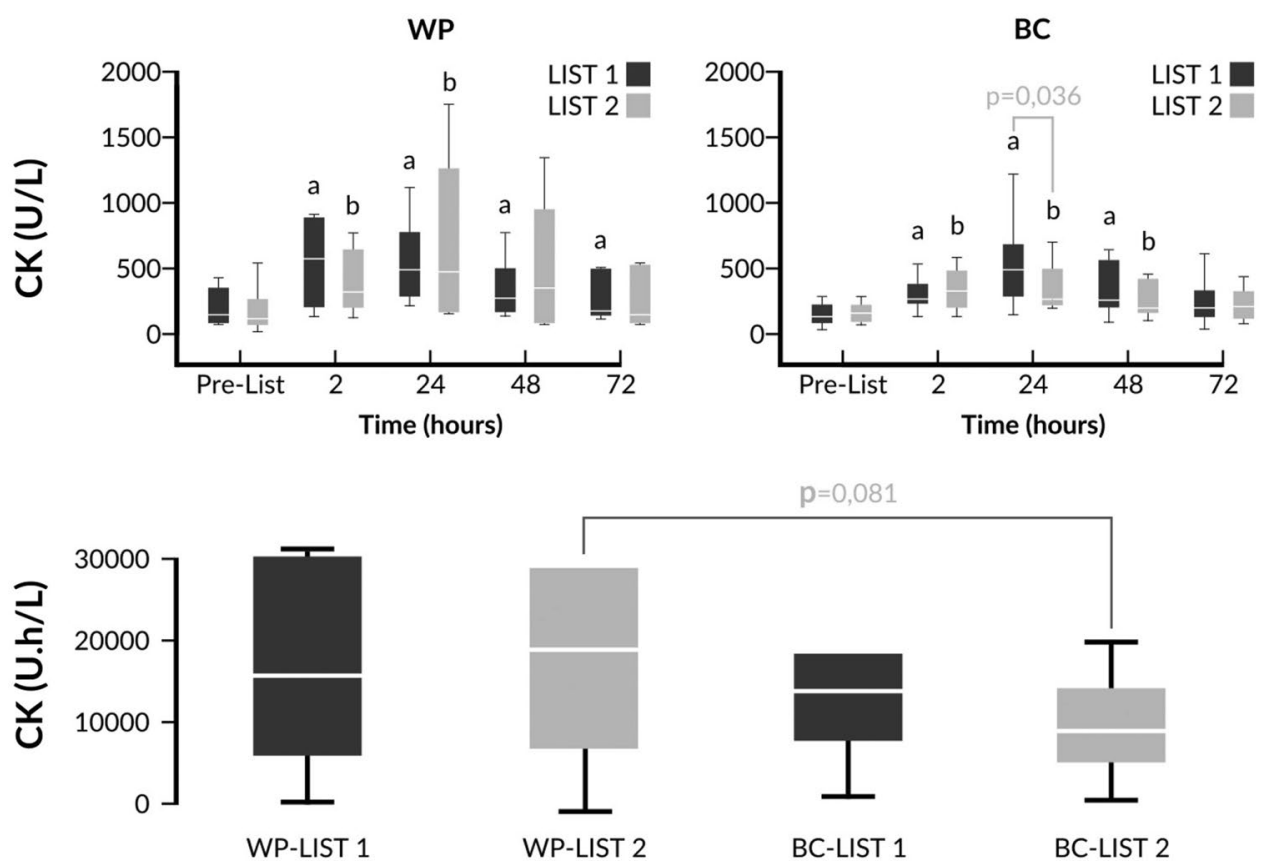

WP-LIST 2

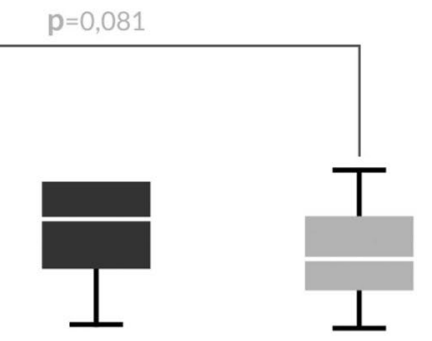

Fig. 4 Changes of $\mathrm{CK}$ and comparison of iAUCs in response to LIST pre- and post-supplementation. ${ }^{a} p<0.05$ vs pre-LIST 1 values, ${ }^{\mathrm{b}} p<0.05$ vs pre-LIST 2 values. CK and iAUC values were $\log$ transformed prior to ANOVA analysis. WP LIST 1 vs WP LIST 2 (two-way ANOVA main effects): $p$ (trial) $=0.833$; $p($ time $) \leq 0.001 ; p($ trial $\times$ time $)=0.743$. BC LIST 1 vs BC LIST 2 (two-way ANOVA main effects): $p$ (trial) $=0.799 ; \mathrm{p}$ (time $) \leq 0.001$; $p($ trial $\times$ time $)=0.056$. WP LIST 1 vs BC LIST 1 (two-way ANOVA main effects): $p$ (intervention) $=0.492 ; p$ (time) $\leq 0.001$; $\mathrm{p}($ intervention $\times$ time $)=0.527$. WP LIST 2 vs BC LIST 2 (twoway ANOVA main effects): $p$ (trial $)=0.671 ; p$ (time) $\leq 0.001$; $p($ trial $\times$ time $)=0.246$
Fig. 5 Changes of CRP and comparison of iAUCs in response to LIST preand post-supplementation. ${ }^{\mathrm{a}} p<0.05$ vs pre-LIST 1 values, ${ }^{\mathrm{b}} p<0.05$ vs pre-LIST 2 values. CRP and iAUC values were log transformed prior to ANOVA analysis. WP LIST 1 vs WP LIST 2 (twoway ANOVA main effects): $p($ trial $)=0.632 ; p$ (time $) \leq$ $0.001 ; p($ trial $\times$ time $)=0.663$.

BC LIST 1 vs BC LIST 2 (two-way ANOVA main effects $): \mathrm{p}($ trial $)=0.544 ; p$ (time) $\leq 0.001 ; p($ trial $\times$ time $)=0.014$. WP LIST 1 vs BC LIST 1 (two-way ANOVA main effects $): p($ intervention $)=0.417$; $p$ (time) $\leq 0.001$;

$p($ intervention $\times$ time $)=0.615$.

WP LIST 2 vs BC LIST 2

(two-way ANOVA main effects $): p($ trial $)=0.717$; $p$ (time) $\leq 0.001$; $\mathrm{p}($ trial $\times$ time $)=0.038$
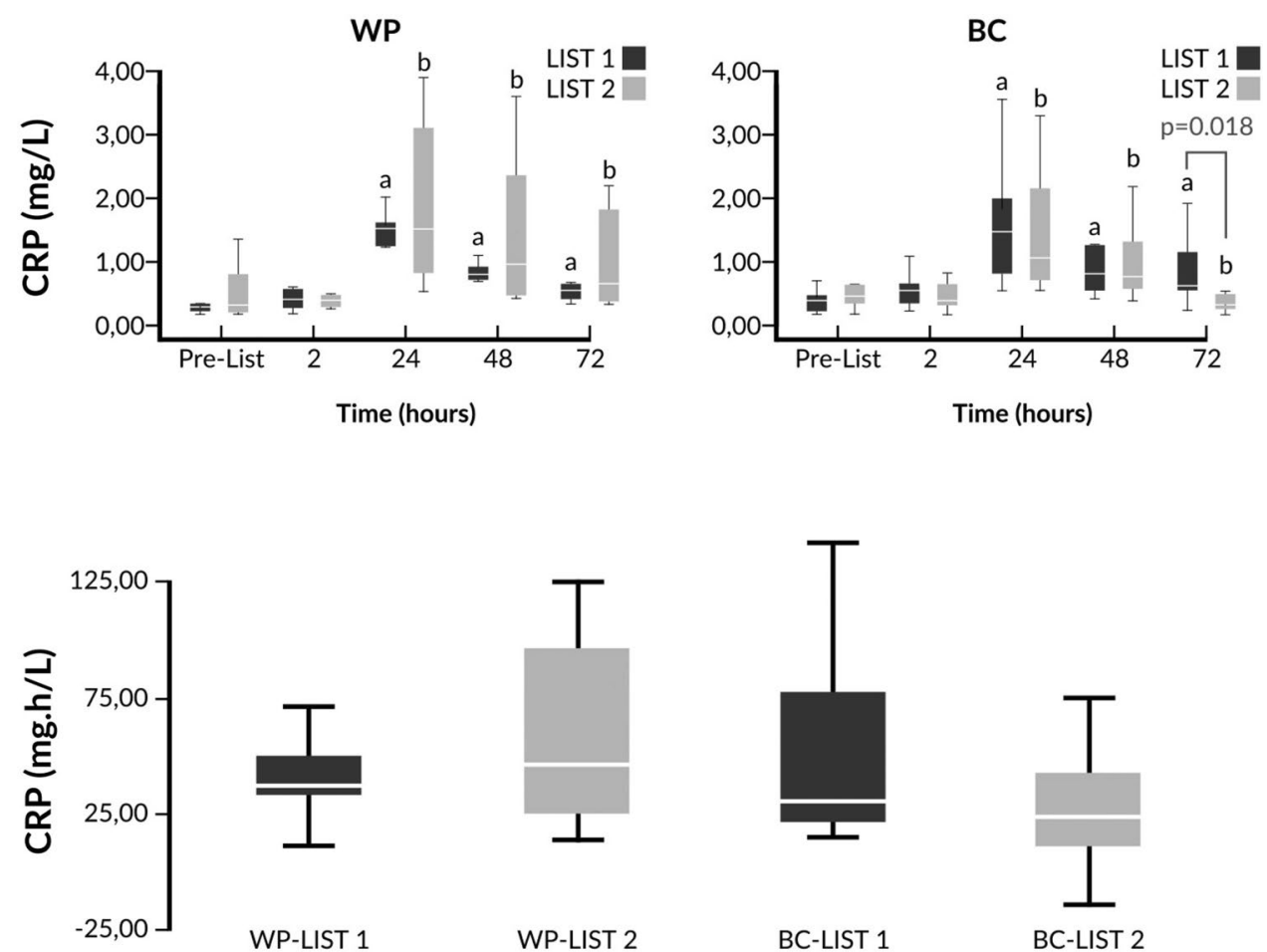

BC-LIST 2 

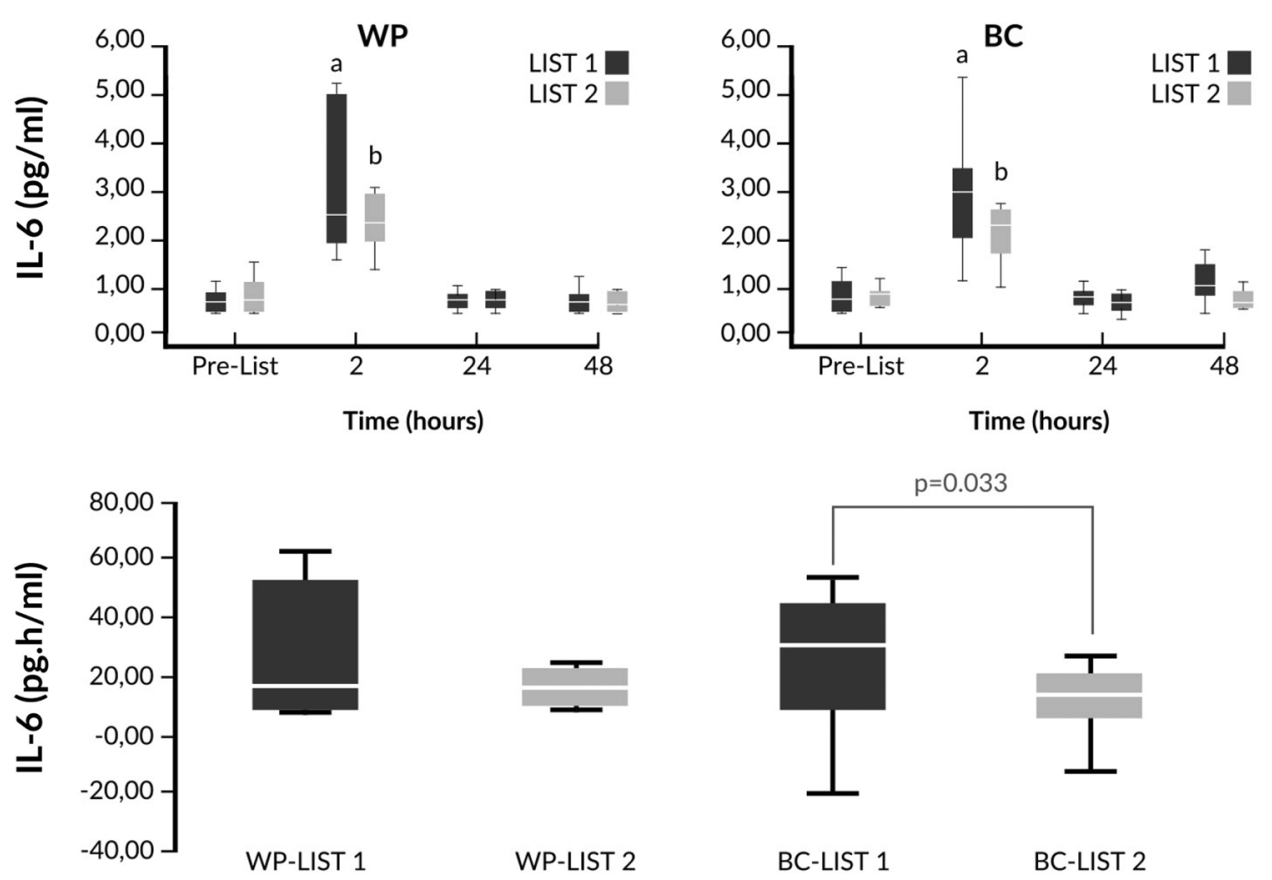

Fig. 6 Changes of IL-6 and comparison of iAUCs in response to LIST pre- and post-supplementation. ${ }^{\mathrm{a}} p<0.05$ vs pre-LIST 1 values, ${ }^{\mathrm{b}} p<0.05$ vs pre-LIST 2 values. IL-6 and iAUC values were $\log$ transformed prior to ANOVA analysis. WP LIST 1 vs WP LIST 2 (two-way ANOVA main effects): $p$ (trial) $=0.853$; $p($ time $) \leq 0.001 ; p($ trial $\times$ time $)=0.529$. BC LIST 1 vs BC LIST 2

When the responses of the biochemical markers were compared between pre- vs post-supplementation LISTs, we observed a non-significant trend for lower elevations of CK activity post-LIST 2 compared to post-LIST 1 only in the BC group. This trend reached statistical significance $24 \mathrm{~h}$ after LIST (Fig. 4). A direct comparison between WP and $\mathrm{BC}$ after the supplementation demonstrated a trend for a lower iAUCs in the $\mathrm{BC}$ group compared to the WP group after LIST 2 ( $p=0.081)$ (Fig. 4).

A significant time $\times$ trial interaction for CRP kinetics was observed only in the $\mathrm{BC}$ group without any significant trial effect (Fig. 5). Lower CRP values were observed at $72 \mathrm{~h}$ after LIST 2 compared to LIST 1 only in the BC group. No significant difference between the iAUCs of the trials was found (Fig. 5). When ANOVA analysis was conducted with the \% changes of CRP values (data not shown) significant differences were observed between BC LIST 1 and BC LIST 2 ( $p$ for trial $=0.038$ ) and between WP LIST 2 and BC LIST 2 ( $p$ for intervention $=0.031)$.

Finally, significantly lower increments of IL-6 were observed in LIST 2 compared to LIST 1 only in the BC group when we compared the iAUCs (Fig. 6) and the percent changes from baseline $2 \mathrm{~h}$ after LIST [LIST 1: $351 \%$ (243-601\%); LIST 2: 254\% (178-339\%), $p=0.028]$.

(two-way ANOVA main effects): $p$ (trial) $=0.445 ; p$ (time) $\leq 0.001$; $\mathrm{p}($ trial $\times$ time $)=0.218$. WP LIST 1 vs BC LIST 1 (two-way ANOVA main effects): $p$ (intervention $)=0.302 ; p$ (time $) \leq 0.001$; $p($ intervention $\times$ time $)=0.392$. WP LIST 2 vs BC LIST 2 (twoway ANOVA main effects): $p$ (trial $)=0.757 ; p$ (time) $\leq 0.001$; $p($ trial $\times$ time $)=0.447$

\section{Discussion}

The present study aimed to investigate the ability of a longterm, low-dose BC supplementation to enhance the recovery process of soccer players after a simulated soccer match (LIST) by alleviating EIMD symptoms. The outcomes of the study demonstrate a trend for $\mathrm{BC}$ supplementation to maintain performance (SQJ decline) and attenuate postexercise increments of CRP, CK and IL-6 in contrast to WP supplementation.

LIST protocol is a well-established, soccer simulation protocol able to induce similar responses to a typical soccer match but in a more controlled fashion [19, 20]. For this reason, LIST has been widely used in supplementation studies which were mainly focused on the potential ergogenic effect in exercise performance [10, 23] fuel replenishment [24] and fluid balance [25]. On the other hand, only few supplementation studies have implemented LIST or other simulated soccer pattern activities $[6,26]$ and real soccer matches [8] to examine the alleviation of EIMD on clinical and biochemical features. Compared to other studies we adopted a relatively long-term ( $>4$ weeks) and lowdose (3.2 g/day vs $20-60 \mathrm{~g} /$ day in other studies) supplementation protocol where the efficacy of the supplementation 
was assessed in a repeated measures design. For the dosage quantity we decided that it should be close enough to the recommendations of the company $(800 \mathrm{mg} /$ day $)$. We speculate that the high bioavailability of $\mathrm{BC}$ in combination with the high quality would be enough to modulate post-exercise kinetics. Despite WADA's statement that "Colostrum is not prohibited per se, however it contains certain quantities of IGF-1 and other growth factors which are prohibited and can influence the outcome of anti-doping tests" (https:// www.wada-ama.org/en/questions-answers/prohibitedlist\#item-388) it is unlikely that our dose scheme could elicit a positive doping test result. The study of Kuipers et al. clearly showed that a 20 -fold higher supplementation dose than ours ( $60 \mathrm{~g} /$ day of BC powder), could not change blood IGF-I or IGF binding protein-3 levels and does not elicit positive results on drug tests [27].

In order to attenuate the high inter-individual variability of such protocols, we compare the response of the same volunteer to LIST pre- and post-supplementation. Moreover, for the simulation of real field settings we incorporated the experimental period during the in-season soccer period. Subjects were committed to a typical soccer training microcycle where a team trains on average $>6$ times and plays one official game per week. During the period of 3 days preceding and 4 days following the LIST, the training load was fixed and controlled by specific training instructions made by our team (Fig. 2). Considering the training background and the engagement in daily training and weekly soccer team routine, we assumed that the repeated bout effect would not be an issue in our experimental conditions.

Our LIST protocol induced similar changes to performance, biochemical and hematological markers of EIMD compared to other studies [1, 28, 29]. In contrast to other similar studies we estimated PMS separately in five leg muscle groups. Due to the eccentric nature of the LIST protocol, a significant increase of perceived muscle pain was observed in all muscle groups but was more pronounced in knee extensors and flexors since they receive the majority of the work load during the acceleration and decelerations phase. Especially the knee flexors contract eccentrically during soccer actions such as kicking, sprinting or changing of directions in order to decelerate thigh and leg movements prior to foot landing [30].

In agreement to our study, the presence of leukocytosis, immediately post-exercise, has also been observed after both LIST and soccer matches although our study showed in a more emphatic way that this leukocytosis is attributed to a dramatic increase of granulocytes, despite a concomitant decrease of lymphocytes $2 \mathrm{~h}$ post-LIST. Similar acute neutrophilia and lymphopenia was observed after a soccer match in both male [31] and female players [32] while other studies were unable to observe this pattern [26, 33]. The main mechanism underlying the exercise-induced lymphopenia is the egress of certain lymphocyte subtypes (NK cells, $\gamma \delta$ Tcells, $\mathrm{CD} 8^{+} \mathrm{T}$ cells) from the blood to peripheral tissues under the influence of glucocorticoids while lymphocyte apoptosis seems to be of minor importance for the observed lymphopenia [34].

Finally, post-exercise elevations of EIMD biochemical marker were in accordance with similar studies after both LIST [7, 28] and soccer protocols [1, 35, 36]. However, the magnitude of the CK increase was lower in our study compared to others $[7,28]$ probably because our volunteers were soccer players, therefore accustomed to the eccentric loads of LIST, especially since the experimental phase took place during the competitive period of the year. Similar CK increments were observed in the work of [3] where volunteers were also competitive soccer players in full training and match schedule.

Both LIST and real soccer matches have been previously used by research to assess the potential beneficial effect of dietary supplements on exercise performance of soccer players. However, studies investigating the ability of supplements to alleviate symptoms of EIMD are scarce and give contradictory results due to different methodological procedures, dose schemes and biochemical measurements. Supplementation with vitamin C [37], a mixture of antioxidant vitamins [7], beta-alanine [10] and green tea polyphenols [38] could not attenuate clinical and biochemical symptoms of EIMD after LIST or similar intermittent tests. On the other hand, DHA supplementation showed a beneficial effect on oxidative stress and inflammatory response after an acute exercise session in soccer players [11]. It is therefore obvious that dietary supplementation in soccer players has not been studied thoroughly and the supplements used until now are incapable of exerting protection against EIMD. This is probably because most of them contain one or few active ingredients with a specific action (e.g., radical scavenging activity), while EIMD is a multifactorial process.

On the other hand, BC is the only naturally produced multi-ingredient supplement. It contains a milieu of bioactive nutrients and microconstituents with antimicrobial and immunomodulatory properties mainly in the alimentary tract, such as lactoferrin, lactoperoxidases, lysozyme, IGF-1/2, peptides and oligosaccharides [14, 39]. The effect of BC supplementation on several aspects of exercise performance and body composition has been extensively studied before; however, the results are contradictory [13]. This can be mainly due to differences in the experimental design, source and composition of $\mathrm{BC}$, dosage schemes and subject's fitness level and training background. However, promising results were obtained when $\mathrm{BC}$ was studied for its ability to attenuate post-exercise immune suppression which is achieved through several mechanisms that include restriction of post-exercise decrements of $\operatorname{IgG} 2$ and 
salivary IgA and lysozyme [40, 41], neutrophils degranulation [40] and protection against exercise-induced gut hyper-permeability [17]. However, the efficiency of BC to protect against EIMD symptoms in soccer players has not been studied before although indirect evidences imply that $\mathrm{BC}$ may act as a recovery enhancer. Brinkworth et al. have shown that BC supplementation to active males $(60 \mathrm{~g} /$ day, 8 weeks) is able to improve performance in an exercise bout 20 min after an initial bout of incremental running exercise to exhaustion [16]. A lower dose of BC (10 g/ day, 5 weeks) was also able to reduce fatigue after a 5 day high intensity training period in highly trained cyclists [42]. The mechanisms underlying the ability of $\mathrm{BC}$ to affect the recovery process after one bout of exercise are still speculative. BC supplementation can increase resting levels of essential and branched-chain amino acids [43] which promote skeletal muscle synthesis and attenuate muscle damage [44]. Moreover, an animal model has shown that $\mathrm{BC}$ in the absence of exercise is able to lower lipid hydroperoxides, increase the specific activity of superoxide dismutase and increase total antioxidant capacity in muscle tissues of rats. When supplemented along with exercise it was able to attenuate exercise-induced increases of lipid hydroperoxides and xanthine oxidase and exercise-induced decreases of SOD and TAC [18]. Finally, BC is able to protect epithelial integrity and attenuate gut permeability partly by reducing temperature-induced apoptosis of epithelial cells and induction of the cytoprotective heat-shock proteins [17]. If similar mechanism were able to function in muscle tissues then they could favorably modulate EIMD. In contrast to other studies, we decided to proceed with a lowdose and relatively long-term dosage scheme which can be applied in real field settings, i.e., during the in-season soccer period.

The results of this study demonstrated the ability of BC supplementation, in contrast to WP supplementation, to attenuate post-LIST increments of CK, CRP and IL-6 accompanied by an improvement of SQJ decrements. BC could not significantly affect PMS, leukocyte counts, MICV, and CMJ kinetics, but instead it had a significant beneficial effect in exercise performance taking into account the improvement in SQJ. It should be mentioned that post-supplementation pre-LIST indices were not affected by BC or WP indicating that BC effects could only be attributed to changes in the post-exercise plasticity of the subject's muscle and inflammatory responses. The greater susceptibility of SQJ to BC supplementation is in accordance with the results of Byrne et al. who found that SQJ performance was affected to a greater extent after an eccentric protocol than the CMJ [45]. These results suggest that the stretch-shortening cycle used in the CMJ possibly masks the negative performance effects associated with the exercise-induced muscle damage. Perhaps the SQJ is more susceptible to the inflammatory response accompanying EIMD than CMJ, which is under the influence of a more complex control and governed by advanced neuromuscular and proprioceptive biofeedback mechanisms. Under this perspective, the mild anti-inflammatory properties of $\mathrm{BC}$ may more strongly affect SQJ than CMJ.

It is tempting to speculate that $\mathrm{BC}$, by attenuating muscle damage (CK and IL-6) can lead to a lower systemic inflammatory response. Whether this attenuation originates from a possible stabilization of muscle cells, which can induce a lower inflammatory response or the opposite, is not known. However, the anti-inflammatory properties of $\mathrm{BC}$ have been demonstrated both in cell and chronic inflammation models $[14,39]$, but these models do not necessarily share similar pathophysiological features with the exercise-induced inflammation. Recently, Appukutty and co-workers [18] demonstrated the ability of BC supplementation to protect against exercise-induced oxidative stress in rat muscles in a dose (per $\mathrm{kg}$ of body weight) similar to ours. In addition, recent studies in athletes have shown the ability of BC to attenuate gut permeability after heavy exercise in athletes [17]. It is therefore obvious that due to its multicomponent composition $\mathrm{BC}$ seems to exert pleiotropic beneficial action on post-exercise recovery. Whether these actions can be translated to long-term beneficial actions on recovery after intermittent exercise requires further mechanistic and supplementation studies.

Our study is the first soccer-specific research that tracks EIMD kinetics and incorporates professional and semiprofessional soccer players accustomed to regular soccer eccentric training instead of amateurs and college students unaccustomed to eccentric exercise mode. In addition there was a high homogeneity between our subjects on their basic anthropometric, biochemical and exercise performance features (i.e., $\mathrm{VO}_{2 \max }$, BMI, training background). Another advantage of the experimental design was the implementation of the supplementation phase during the in season period of the professional league. The soccer training micro-cycle per se can produce an additional load on musculoskeletal system of the athlete and an added difficulty for the BC supplementation to overcome. We tried to simulate/ mimic the real soccer field settings even though we knew that regular high-intensity soccer practice, with the cyclic pattern of concentric and eccentric extension/flexion of the knee per se, might alter the athlete performance decline. To overcome the latter, we decide to incorporate a specific training program 3 days pre- and 4 days post-LIST without any additional eccentric load. In addition, we choose functional testing like MICV, SQJ and CMJ that have minimum eccentric load and would not exaggerate or interfere with the EIMD symptoms caused by the LIST per se.

On the other hand, the low number of participants, the nature of the protocol which lacks soccer-specific 
movements like tackling or shooting, the limited range of functional tests, the use of maximal isometric strength of the knee extensors instead of knee flexors which are more susceptible to soccer eccentric movement pattern and the use of just one dose of $\mathrm{BC}$ supplementation are among the limitations of the study.

In conclusion, the LIST protocol can induce EIMD and performance decrements in soccer players. EIMD and performance following LIST intervention may positively be affected by a low-dose administration of $\mathrm{BC}$ in soccer players. Larger studies are needed to confirm the ability of bovine colostrum to improve recovery after a soccer game.

Acknowledgements We wish to thank Antigoni Tsiafitsa for her excellent technical support and all the volunteers for their participation.

\section{Compliance with ethical standards}

Funding The study was partially funded by LR Health and Beauty Systems.

Conflict of interest Yiannis Kotsis works as a part-time scientific consultant for LR Health and Beauty Systems.

Open Access This article is distributed under the terms of the Creative Commons Attribution 4.0 International License (http:// creativecommons.org/licenses/by/4.0/), which permits unrestricted use, distribution, and reproduction in any medium, provided you give appropriate credit to the original author(s) and the source, provide a link to the Creative Commons license, and indicate if changes were made.

\section{References}

1. Nédélec $\mathrm{M}$, McCall A, Carling $\mathrm{C}$, Legall $\mathrm{F}$, Berthoin $\mathrm{S}$, Dupont G (2012) Recovery in soccer: part I-post-match fatigue and time course of recovery. Sports Med 42:997-1015. doi:10.2165/11635270-000000000-00000

2. Rampinini E, Sassi A, Azzalin A, Castagna C, Menaspa P, Carlomagno D, Impellizzeri FM (2010) Physiological determinants of Yo-Yo intermittent recovery tests in male soccer players. Eur J Appl Physiol 108:401-409. doi:10.1007/s00421-009-1221-4

3. Mohr M, Draganidis D, Chatzinikolaou A, Barbero-Álvarez JC, Castagna C, Douroudos I, Avloniti A, Margeli A, Papassotiriou I, Flouris AD, Jamurtas AZ, Krustrup P, Fatouros IG (2016) Muscle damage, inflammatory, immune and performance responses to three football games in 1 week in competitive male players. Eur J Appl Physiol 116:179-193. doi:10.1007/ s00421-015-3245-2

4. Carling C, Gregson W, McCall A, Moreira A, Wong del P, Bradley PS (2015) Match running performance during fixture congestion in elite soccer: research issues and future directions. Sports Med 45:605-613. doi:10.1007/s40279-015-0313-z

5. Nedelec M, McCall A, Carling C, Legall F, Berthoin S, Dupont G (2013) Recovery in soccer : part ii-recovery strategies. Sports Med 43:9-22. doi:10.1007/s40279-012-0002-0

6. Cox G, Mujika I, Tumilty D, Burke L (2002) Acute creatine supplementation and performance during a field test simulating match play in elite female soccer players. Int $\mathbf{J}$ Sport Nutr Exerc Metab 12:33-46

7. Bailey DM, Williams C, Betts JA, Thompson D, Hurst TL (2011) Oxidative stress, inflammation and recovery of muscle function after damaging exercise: effect of 6-week mixed antioxidant supplementation. Eur J Appl Physiol 111:925-936. doi:10.1007/s00421-010-1718-x

8. Tauler P, Ferrer MD, Sureda A, Pujol P, Drobnic F, Tur JA, Pons A (2008) Supplementation with an antioxidant cocktail containing coenzyme Q prevents plasma oxidative damage induced by soccer. Eur J Appl Physiol 104:777-785. doi:10.1007/s00421-008-0831-6

9. Djordjevic B, Baralic I, Kotur-Stevuljevic J, Stefanovic A, Ivanisevic J, Radivojevic N, Andjelkovic M, Dikic N (2012) Effect of astaxanthin supplementation on muscle damage and oxidative stress markers in elite young soccer players. J Sports Med Phys Fit 52:382-392

10. Saunders B, Sale C, Harris RC, Sunderland C (2012) Effect of beta-alanine supplementation on repeated sprint performance during the Loughborough Intermittent Shuttle Test. Amino Acids 43:39-47. doi:10.1007/s00726-012-1268-0

11. Martorell M, Capó X, Sureda A, Batle JM, Llompart I, Argelich E, Tur JA, Pons A (2014) Effect of DHA on plasma fatty acid availability and oxidative stress during training season and football exercise. Food Funct 5:1920-1931. doi: $10.1039 / \mathrm{c} 4$ fo00229f

12. Struff WG, Sprotte G (2007) Bovine colostrum as a biologic in clinical medicine: a review. Part I: biotechnological standards, pharmacodynamic and pharmacokinetic characteristics and principles of treatment. Int J Clin Pharmacol Ther 45:193-202

13. Shing CM, Hunter DC, Stevenson LM (2009) Bovine colostrum supplementation and exercise performance: potential mechanisms. Sports Med 39:1033-1054. doi:10.2165/11317860-000000000-00000

14. Davison G (2012) Bovine colostrum and immune function after exercise. Med Sport Sci 59:62-69. doi:10.1159/000341966

15. Antonio J, Sanders MS, Van Gammeren D (2001) The effects of bovine colostrum supplementation on body composition and exercise performance in active men and women. Nutrition 17:243-247

16. Brinkworth GD, Buckley JD, Bourdon PC, Gulbin JP, David A (2002) Oral bovine colostrum supplementation enhances buffer capacity but not rowing performance in elite female rowers. Int $\mathbf{J}$ Sport Nutr Exerc Metab 12:349-365

17. Marchbank T, Davison G, Oakes JR, Ghatei MA, Patterson M, Moyer MP, Playford RJ (2011) The nutriceutical bovine colostrum truncates the increase in gut permeability caused by heavy exercise in athletes. Am J Physiol Gastrointest Liver Physiol 300:G477-G384. doi:10.1152/ajpgi.00281.2010

18. Appukutty M, Radhakrishnan AK, Ramasamy K, Ramasamy R, Abdul Majeed AB, Noor MI, Safii NS, Koon PB, Chinna K, Haleagrahara N (2012) Colostrum supplementation protects against exercise-induced oxidative stress in skeletal muscle in mice. BMC Res Notes 5:649. doi:10.1186/1756-0500-5-649

19. Nicholas CW, Nuttall FE, Williams C (2000) The Loughborough Intermittent Shuttle Test: a field test that simulates the activity pattern of soccer. J Sports Sci 18:97-104

20. Thompson D, Nicholas CW, Williams C (1999) Muscular soreness following prolonged intermittent high-intensity shuttle running. J Sports Sci 17:387-395

21. Detopoulou P, Nomikos T, Fragopoulou E, Panagiotakos DB, Pitsavos C, Stefanadis C, Antonopoulou S (2009) Lipoproteinassociated phospholipase A2 (Lp-PLA2) activity, plateletactivating factor acetylhydrolase (PAF-AH) in leukocytes and body composition in healthy adults. Lipids Health Dis 8:19. doi:10.1186/1476-511X-8-19 
22. Bosco C, Ito A, Komi PV, Luhtanen P, Rahkila P, Rusko H, Viitasalo JT (1982) Neuromuscular function and mechanical efficiency of human leg extensor muscles during jumping exercises. Acta Physiol Scand 114:543-550

23. Andrade-Souza VA, Bertuzzi R, de Araujo GG, Bishop D, LimaSilva AE (2005) Effects of isolated or combined carbohydrate and caffeine supplementation between 2 daily training sessions on soccer performance. Appl Physiol Nutr Metab 40:457-463. doi:10.1139/apnm-2014-0268

24. Erith S, Williams C, Stevenson E, Chamberlain S, Crews P, Rushbury I (2006) The effect of high carbohydrate meals with different glycemic indices on recovery of performance during prolonged intermittent high-intensity shuttle running. Int J Sport Nutr Exerc Metab 16:393-404

25. Owen JA, Kehoe SJ, Oliver SJ (2013) Influence of fluid intake on soccer performance in a temperate environment. J Sports Sci 31:1-10. doi:10.1080/02640414.2012.720701

26. Naclerio F, Larumbe-Zabala E, Cooper R, Allgrove J, Earnest CP (2015) A multi-ingredient containing carbohydrate, proteins L-glutamine and L-carnitine attenuates fatigue perception with no effect on performance, muscle damage or immunity in soccer players. PLoS One 10:e0125188. doi:10.1371/journal. pone. 0125188

27. Kuipers H, van Breda E, Verlaan G, Smeets R (2002) Effects of oral bovine colostrum supplementation on serum insulin-like growth factor-I levels. Nutrition 18:566-567

28. Leeder J DC, van Someren KA, Gaze D, Jewell A, Deshmukh NI, Shah I, Barker J, Howatson G (2014) Recovery and adaptation from repeated intermittent-sprint exercise. Int J Sports Physiol Perform 9:489-496. doi:10.1123/ijspp.2012-0316

29. Magalhães J, Rebelo A, Oliveira E, Silva JR, Marques F, Ascensão A (2010) Impact of Loughborough Intermittent Shuttle Test versus soccer match on physiological, biochemical and neuromuscular parameters. Eur J Appl Physiol 108:39-48. doi:10.1007/s00421-009-1161-z

30. Draganidis D, Chatzinikolaou A, Avloniti A, Barbero-Álvarez JC, Mohr M, Malliou P, Gourgoulis V, Deli CK, Douroudos II, Margonis K, Gioftsidou A, Flouris AD, Jamurtas AZ, Koutedakis Y, Fatouros IG (2015) Recovery kinetics of knee flexor and extensor strength after a football match. PLoS One 10:e0128072. doi:10.1371/journal.pone.0128072

31. Ascensão A, Rebelo A, Oliveira E, Marques F, Pereira L, Magalhães J (2008) Biochemical impact of a soccer matchanalysis of oxidative stress and muscle damage markers throughout recovery. Clin Biochem 41:841-851. doi:10.1016/j. clinbiochem.2008.04.008

32. Gravina L, Ruiz F, Lekue JA, Irazusta J, Gil SM (2011) Metabolic impact of a soccer match on female players. J Sports Sci 29:1345-1352. doi:10.1080/02640414.2011.597420

33. Ferrer MD, Tauler P, Sureda A, Pujol P, Drobnic F, Tur JA, Pons A (2009) A soccer match's ability to enhance lymphocyte capability to produce ROS and induce oxidative damage. Int $\mathrm{J}$ Sport Nutr Exerc Metab 19:243-258

34. Peake JM, Neubauer O, Walsh NP, Simpson RJ (2016) Recovery of the immune system after exercise J Appl Physiol (1985) 1:jap.00622.2016. doi:10.1152/japplphysiol.00622.2016

35. Ispirlidis I, Fatouros IG, Jamurtas AZ, Nikolaidis MG, Michailidis I, Douroudos I, Margonis K, Chatzinikolaou A, Kalistratos E, Katrabasas I, Alexiou V, Taxildaris K (2008) Time-course of changes in inflammatory and performance responses following a soccer game. Clin J Sport Med 18:423-431. doi:10.1097/ JSM.0b013e $3181818 \mathrm{e} 0 \mathrm{~b}$

36. Souglis A, Bogdanis GC, Giannopoulou I, Papadopoulos Ch, Apostolidis N (2015) Comparison of inflammatory responses and muscle damage indices following a soccer, basketball, volleyball and handball game at an elite competitive level. Res Sports Med 23:59-72. doi:10.1080/15438627.2014.975814

37. Thompson D, Williams C, Kingsley M, Nicholas CW, Lakomy HK, McArdle F, Jackson MJ (2001) Muscle soreness and damage parameters after prolonged intermittent shuttle-running following acute vitamin C supplementation. Int J Sports Med 22(1):68-75

38. Jówko E, Sacharuk J, Balasinska B, Wilczak J, Charmas M, Ostaszewski P, Charmas R (2012) Effect of a single dose of green tea polyphenols on the blood markers of exercise-induced oxidative stress in soccer players. Int J Sport Nutr Exerc Metab 22:486-496

39. Rathe M, Müller K, Sangild PT, Husby S (2014) Clinical applications of bovine colostrum therapy: a systematic review. Nutr Rev 72:237-254. doi:10.1111/nure

40. Davison G, Diment BC (2010) Bovine colostrum supplementation attenuates the decrease of salivary lysozyme and enhances the recovery of neutrophil function after prolonged exercise. Br J Nutr 103:1425-1432. doi:10.1017/S0007114509993503

41. Crooks CV, Wall CR, Cross ML, Rutherfurd-Markwick KJ (2006) The effect of bovine colostrum supplementation on salivary $\operatorname{IgA}$ in distance runners. Int J Sport Nutr Exerc Metab $16: 47-64$

42. Shing CM, Jenkins DG, Stevenson L, Coombes JS (2006) The influence of bovine colostrum supplementation on exercise performance in highly trained cyclists. Br J Sports Med 40:797-801

43. Mero A, Nykänen T, Keinänen $\mathrm{O}$, Knuutinen J, Lahti K, Alen M, Rasi S, Leppäluoto J (2005) Protein metabolism and strength performance after bovine colostrum supplementation. Amino Acids 28:327-335

44. Shimomura Y, Yamamoto Y, Bajotto G, Sato J, Murakami T, Shimomura N, Kobayashi H, Mawatari K (2006) Nutraceutical effects of branched-chain amino acids on skeletal muscle. J Nutr 136:529S-532S

45. Byrne C, Eston R (2002) Maximal-intensity isometric and dynamic exercise performance after eccentric muscle actions. J Sports Sci 20:951-959 